\section{SINGLE-MODE FIBRE Y-JUNCTION BEAM-SPLITTER}

Indexing terms: Optical fibres, Optical connectors and couplers

The fabrication, operation and performance of a wavelengthindependent single-mode fibre beam-splitter is discussed. Equal power splitting over an indefinitely broad spectral range is possible with this device, which has a tapered Yjunction configuration.

Introduction: There have recently been several proposals for wavelength-independent and wavelength-insensitive singlemode fibre couplers and splitters. ${ }^{1,2}$ Such devices are desirable because of their reproducible characteristics, and should be useful for wavelength-multiplexed and spectral measurement systems.

In this letter we discuss a new structure based on a tapered Y-junction configuration. This structure has previously been proposed for multimode fibre beam-splitters. ${ }^{3,4}$ The behaviour of single-mode tapers and couplers ${ }^{5}$ allows us to extend the use of the $\mathrm{Y}$-junction to single-mode fibre technology.

Structure: The basic structure of the beam-splitter is shown in Fig. 1a. The device has three ports, one input and two output ports, arranged as a ' $Y$ '.

A single-mode fibre taper, which forms the stem of the ' $Y$ ', is spliced to a matched half of a circularly fused tapered coupler which forms the fork.

Principles of operation: Single-mode fibre tapers and couplers are now known to be essentially cladding mode devices. ${ }^{6}$ The coupler requires the equal excitation of the two lowest-order

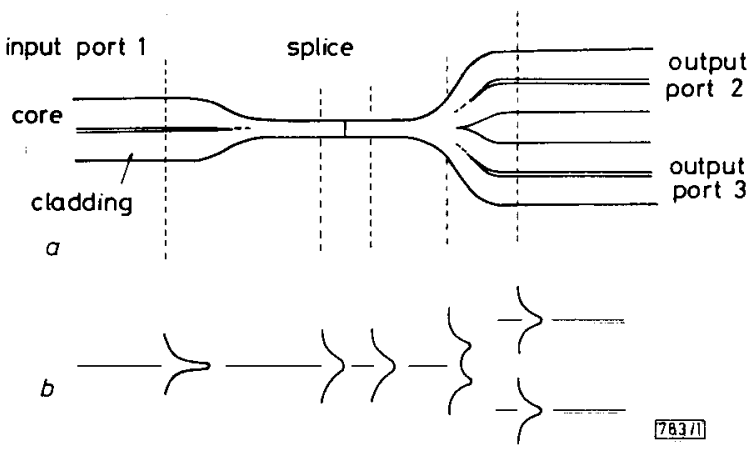

Fig. 1 Schematic diagrams of (a) single-mode wavelength-independent $Y$-junction beam-splitter and (b) $L P_{01}$ field evolution

( $L P_{01}$ and $\left.L P_{11}\right)$ modes of the cladding/air waveguide formed in the coupler waist. Coupling results from the interference between these two modes. The output power splitting ratio depends on the length, the cross-sectional size and shape of the coupling region, the external refractive index and the polarisation state and wavelength of the input light.

The Y-junction approach eliminates all these dependencies by exciting only one cladding mode at the waist of the device. Its operation is similar to that of the mode transformer of Mortimore and Wright ${ }^{7}$ used in the production of low-loss splices between dissimilar fibres.

The fundamental $\left(\mathrm{LP}_{01}\right)$ mode, which is initially strongly confined to the core of the input fibre, spreads out as it propagates along the input taper. At some point along the taper the field becomes bound at the strongly guiding cladding/air boundary and, provided that the core is now sufficiently small, this boundary then dominates the guidance of the mode. The transition is a gradual one, but with standard fibre operating just below second-mode cutoff a useful rule of thumb is to assume cladding mode behaviour after a taper ratio of about $3: 1$.

The light now propagates across the splice point with little loss, since the modal fields on either side of this point are reasonably well matched. The excitation symmetry in a perfect Y-junction ensures that there is no excitation of the LP $P_{11}$ mode and hence that there is no wavelength dependence in the device. The adiabatically increasing cores at the tork side of the junction now guide power equally into the two output ports for all wavelengths, irrespective of external index and polarisation. The $\mathbf{L} \mathbf{P}_{01}$ field evolution in the tapered $\mathbf{Y}$ junction is shown schematically in Fig. $1 b$.

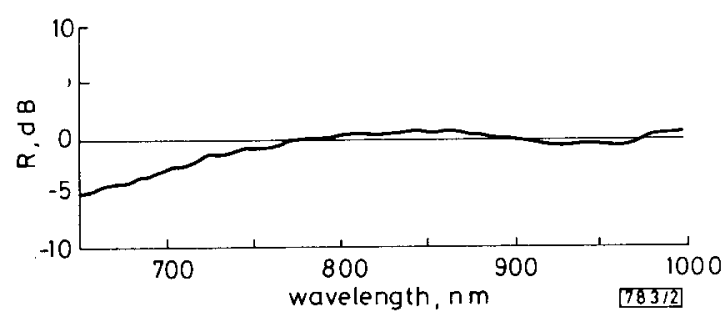

Fig. 2 Wavelength response of beam-splitter

Splitting ratio is defined as $R=10 \log _{10}\left(P_{2} / P_{3}\right)$, where $P_{2}=$ power in port 2 and $P_{3}=$ power in port 3

Results and discussion: The wavelength response from 650 to $1000 \mathrm{~nm}$ for one of our beam-splitters is shown in Fig. 2. This Y-junction had an excess loss of $1.5 \mathrm{~dB}$. The fibre had a numerical aperture of $0 \cdot 19$, a diameter of $80 \mu \mathrm{m}$ and a secondmode cutoff wavelength of $650 \mathrm{~nm}$. The residual wavelength
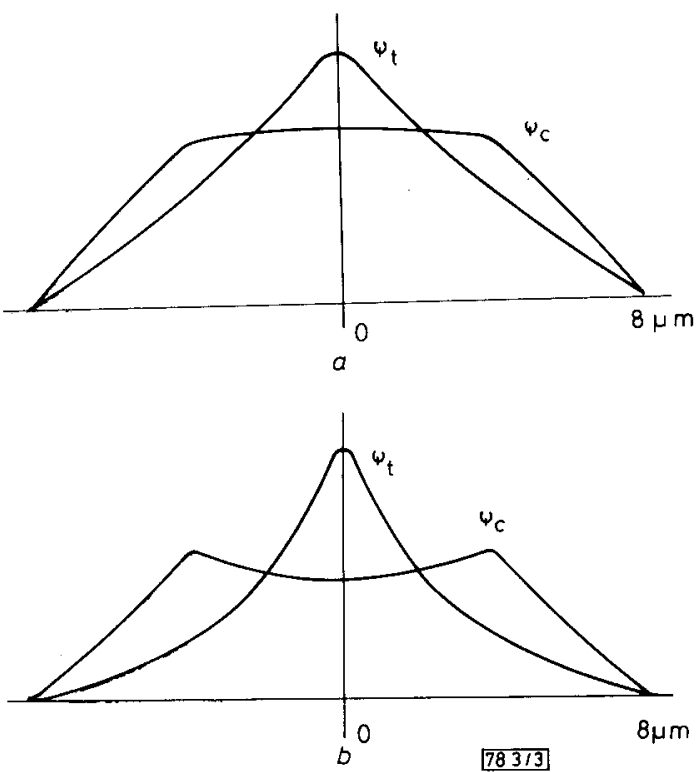

Fig. 3 Calculated $L P_{01}$ modal field distributions on either side of splice point for fibre taper ratio $T_{R}=5$ and $N A$ of $(a) 0 \cdot 1$ and $(b) 0.2$

$\psi_{t}$ is field in taper and $\psi_{c}$ is field in half-coupler

response is caused by a limited excitation of the $L P_{11}$ mode. This is believed to be due to a slight transverse offset or tilt at the splice point.

The excess loss of the Y-junction depends on several factors, notably: the adiabaticity of the input and output transitions; the circularity of the half-coupler waist; the extent of diameter mismatching at the splice point; the residual effects of the fibre cores on the matching of the field distributions. This last factor sets a fundamental limit on the device performance.

To quantify this fundamental limit in excess loss, an exact study of field overlap between two cladding waveguides with the cores still accounted for was undertaken. The calculations were based on slab waveguides with starting outside dimensions similar to the fibre used in the experiment.

Fig. 3 shows the fields on either side of the splice point for two different values of core NA for a given taper ratio. Clearly the field match is better for the lower NA fibre.

Fig. 4 shows the calculated loss as a function of taper ratio for two different values of core NA. Field matching also improves rapidly with taper ratio, irrespective of core NA.

The limit to taper ratio is the ability to splice such small fibres together. With our apparatus we could, with care, splice tapers down to $15 \mu \mathrm{m}$.

In this letter we have considered a three-port device where the power is divided equally between two output ports. 


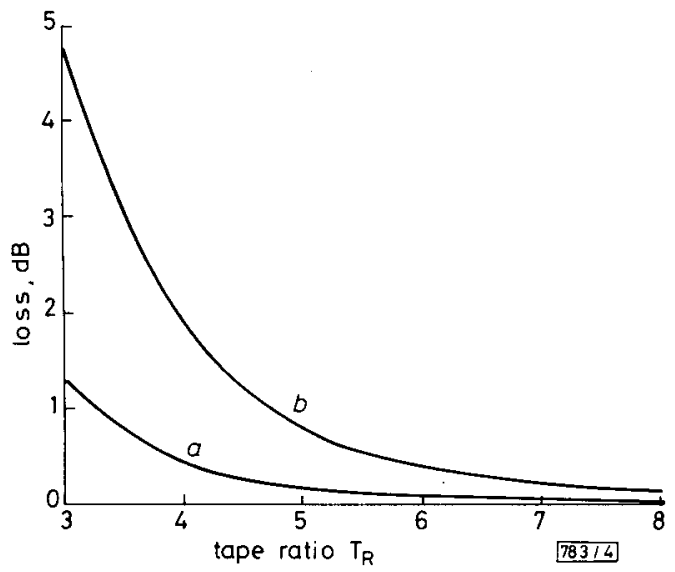

Fig. 4 Calculated $Y$-junction loss as a function of taper ratio $T_{R}$ for fibres with core $N A$ of $(a) 0.1$ and $(b) 0.2$

However, there is no restriction in principle as to the number of output ports for which equal power splitting will still occur. The input and output fibres will normally be identical, although in general the input fibre can be completely different (i.e. different diameter and refractive index).
Acknowledgment: This work was supported by the UK SERC.

\section{J. D. MINELLY \\ C. D. HUSSEY}

21st July 1987

Department of Electronics \& Computer Science

University of Southampton

Southampton SO9 5NH, United Kingdom

\section{References}

1 MORTIMORE, D. B.: 'Wavelength-flattened fused couplers', Electron. Lett., 1985, 21, pp. 742-743

2 QIAN, J.: 'A new idea for wavelength-insensitive fibre couplers'. IEE colloquium on advanced fibre waveguide devices, 20 May 1986, IEE colloquium digest 1986/79

3 EMKEY, W. L.: 'Non tapered fused fibre couplers'. Proc. 8th conference on optical fibre communications, 1985, pp. 108-109

4 FioRina, L., MEZzetTI, S., and SVELto, F.: 'Low-loss Y-coupler for multimode single fibres', Electron. Lett., 1978, 14, pp. 808-809

5 PAYNE, F. P., HUSSEY, C. D., and YATAKI, M. S.: 'Polarisation analysis of strongly fused and weakly fused tapered couplers', ibid., 1985, 21, pp. 561-563

6 BURRES, J., LACROUX, S., and LAPPIERRE, J.: 'Analyses d'un coupleur bidirectionnel a fibres optiques monomode fusionnees', Appl. Opt., 1983, 22, pp. 1918-1922

7 MORTIMORE, D. B., and WRIGHT, J. v.: 'Low-loss joints between dissimilar fibres by tapering fusion splices', Electron. Lett., 1986, 22, pp. 318-319 